\title{
ANALISIS DEL PROGRAMA DE URBANIZACIÓN “MI TIERRA, MI CASA" EN LA CIUDAD DE ROSARIO
}

\author{
Dra. Arq. Cintia Ariana Barenboim \\ CURDIUR (CONICET), Facultad de Arquitectura (UAI) e Ingeniería Civil (UNR) \\ arq.barenboim@gmail.com
}

\section{RESUMEN}

Durante las últimas décadas las condiciones para acceder a una vivienda en las ciudades argentinas han sido difíciles. El precio de los terrenos e inmuebles es cada vez mayor principalmente por la demanda extra de vivienda que lejos de tener un fin social tuvo un fin financiero, como inversión y/o resguardo de capital.

En este contexto, el desafío del Gobierno Provincial fue elaborar el Programa "Mi Tierra, Mi Casa", promoviendo el acceso al suelo urbanizado para los sectores sociales medios y medio-bajos que no poseen lote propio 0 vivienda, a precios inferiores del mercado inmobiliario.

En primer lugar se describe concisamente el Programa (objetivos, beneficiarios, línea de crédito y articulación con otros programas); luego se identifica el alcance en la ciudad de su origen Rosario (ubicación, cantidad de lotes, prototipos, inserción en el tejido urbano) y por último, se evalúa los cambios realizados en el programa y las acciones ejecutadas.

Palabras claves: financiamiento público, clase media - baja, acceso a suelo urbanizado, vivienda propia.

\begin{abstract}
During the last decades the conditions to access a home in Argentine cities have been difficult. The price of land and real estate is increasing, mainly due to the extra demand for housing that, far from having a social purpose, had a financial purpose, such as investment and / or capital protection.

In this context, the challenge of the Provincial Government was to develop the "My Land, My House" Program, promoting access to urbanized land for medium and low-middle social sectors that do not own their own lot or housing, at lower prices of the real estate market .

First, the Program is briefly described (objectives, beneficiaries, line of credit and articulation with other programs); then the scope in the city of its Rosario origin is identified (location, number of lots, prototypes, insertion in the urban fabric) and finally, the changes made to the program and the actions carried out are evaluated.
\end{abstract}

Key words: public financing, middle class - low, access to urbanized land, own housing. 


\section{INTRODUCCIÓN}

A pesar de que durante la última década hubo una mejora de los indicadores económicos y sociales, el incremento de la construcción y la expansión de la oferta residencial, las condiciones para acceder a una vivienda formal en las grandes ciudades argentinas (Buenos Aires, Córdoba y Rosario) continuaron siendo difíciles (Barenboim, 2017:131).

La vivienda lejos de tener un fin social tuvo un fin netamente financiero como resguardo de capital, produciéndose en algunos casos edificaciones sin ningún tipo de planificación de la forma urbana. Por lo general las unidades, no se produjeron ni se compraron como un bien de uso, sino como un activo que soportaba el valor de los ahorros.

Esta demanda extra en el mercado de compra y venta de inmuebles, sumado a la depreciación del dólar y a la inflación monetaria, entre otras variables, según el Centro de Estudios Económico y Sociales Scalabrini Ortiz (2016), encareció las propiedades hasta superar las posibilidades de la mayoría de la población.

Cabe señalar que el soporte y la materia prima de toda edificación es el suelo, siendo el acceso al mismo, por lo general, una de las cuestiones críticas para el acceso a la vivienda. Al respecto, Sciara (2011:5) expresa que: "... el derecho a la vivienda se subsume en el derecho a la ciudad: sin la satisfacción de este, la satisfacción del primero es precaria o imperfecta".

La brecha entre los requerimientos (problema de habitabilidad o falta de vivienda) y la disponibilidad de viviendas adecuadas para un sector de la sociedad, lo que el Celade (2010) denomina como "déficit habitacional", es cada vez mayor y presenta una persistencia crónica en el tiempo.

Particularmente, la clase media y media-baja es uno de los sectores sociales más perjudicados dado que no ha contado con políticas y/o programas de financiamiento público permanentemente. Es por ello que recurren al mercado de alquiler o a la construcción de viviendas propias en la periferia, donde el valor del suelo es menor que en la ciudad. Los sectores bajos, por su parte, acuden a la construcción de viviendas precarias en asentamientos informales, cuando no son absorbidos por los planes sociales del Estado (FONAVI, Hábitat, PROMEBA, entre otros), compitiendo ambos por los espacios vacantes de la ciudad. Por último, la clase alta accede a la vivienda sin ningún tipo de problema, siendo además el sector de mayor inversión inmobiliaria.

Las respuestas del Estado en ciertas ocasiones son de dos maneras: directa a través de la construcción o mejoramiento de la vivienda e indirecta cuando otorga un crédito para construcción, ampliación o compra de la unidad. Barenboim (2017:134) expresa que: "de ningún modo, regula el mercado de suelo urbano, siendo este un elemento central para el acceso al suelo y a la vivienda".

En este contexto, interesa analizar el Programa "Mi Tierra, Mi Casa", el cual promueve el acceso al suelo urbanizado para los sectores sociales medios y medio-bajos que no poseen lote propio o vivienda, a precios inferiores del mercado inmobiliario, instrumentado desde la Secretaría de Estado del Hábitat (SEH) en la provincia de Santa Fe, a partir del año 2012. Esto fue posible dado que va rehabilitando y completando progresivamente grandes áreas del tejido urbano, que tienen todas las facilidades técnicas para extender las infraestructuras y los servicios, logrando además parte del derecho a la ciudad. Trabaja en forma conjunta con la planificación urbana local a partir de la propuesta de cada plan urbano en donde se establece hacia donde puede crecer la ciudad y cuáles son los lugares vacantes a completar.

El área de estudio comprende a la ciudad de Rosario, uno de los sitios más favorecidos por el escenario macroeconómico, principalmente por la renta generada del sector agropecuario volcada en la industria de la construcción, conocida como la "ciudad inmobiliaria" y contrariamente la de mayor déficit habitacional de la provincia. Al respecto, el Consejo Económico y Social (2010), señaló que en Rosario hay 50.000 hogares que tienen necesidades de una nueva vivienda, sea por habitar en edificaciones en malas condiciones o por hacinamiento. La mitad del total, se encuentra en los sectores de ingresos medios y medio-bajos mientras la otra en el segmento de menores recursos. Álvarez del Servicio Público de la Vivienda agrega que: 
... para los sectores bajos es el mismo valor que la Fundación Techo Argentina procuró de aproximadamente 35.600 familias que viven en condiciones inadecuadas por relocalización (vías férreas, zonas inundables), falta de infraestructura básica o mal estado de la vivienda mientras que para los sectores medios, gente buscando su casa propia, este valor podría haber crecido para el año 2017 a un valor aproximado 30.000 familias".

También Rosario es el lugar de origen del Programa en donde el desarrollador público realizo tres urbanizaciones: Ibarlucea, Avellaneda Oeste y Ex. Villa Olímpica. Estas se encuentran dentro de planes espaciales de "parques habitacionales", cuya idea es ordenar los usos y características de edificación para la urbanización de un sector de la ciudad, principalmente destinado a la actividad residencial. Además, implica la provisión de infraestructura y equipamientos, incorporando en algunos casos otros usos de carácter secundarios, como ser el comercial (Barenboim, 2014: 174).

El objetivo principal se centra en analizar la alternativa de financiamiento público de "Mi Tierra, Mi Casa", en cuanto a su contenido e implementación en Rosario. Los objetivos secundarios se refieren a describir concisamente el Programa (objetivos, beneficiarios, modalidad de financiamiento y articulación con otros programas); identificar cuál fue su alcance en la ciudad a través del análisis de las tres urbanizaciones (ubicación, cantidad de lotes e inserción en el tejido urbano) y los prototipos universales diseñados por el Gobierno Provincial. Por último, evaluar los cambios que tuvieron el programa durante su gestión y las acciones ejecutadas en Rosario.

La hipótesis radica en que las nuevas urbanizaciones se encuentran en zonas lejanas, prácticamente en los bordes periurbanos, fomentando el Estado una profunda segmentación socioespacial para los sectores medios y medio-bajos, a pesar de que los beneficiarios logran acceder a la vivienda propia a precios accesibles. En este sentido, la SEH se distingue de las políticas habitacionales tradicionales debido a que evita la especulación inmobiliaria, en donde el Estado es el desarrollador urbano, y establece un modelo de esfuerzo compartido entre el gobierno, empresas, instituciones y beneficiarios en las distintas etapas del proceso. Sin embargo, deja en claro el protagonismo que posee la dimensión espacial en las políticas públicas aplicadas, en materia de localización de conjuntos residenciales, en las últimas décadas.

La investigación se centra en un abordaje analítico con el análisis de contenido de documentos escritos y gráficos como ser:

- $\quad$ No oficiales: trabajos de investigación y artículos periodísticos locales.

- $\quad$ Oficiales: documentos del Programa Mi tierra, Mi casa.

- $\quad$ Planos de los prototipos e Imágenes satelitales de las urbanizaciones.

Además, se recurre a la modalidad interpretativa para la realización de entrevistas semi- estructurada a actores claves, con el propósito de completar la información y conocer la visión e instrumentación la modalidad de financiamiento.

\section{DESCRIPCIÓN DEL PROGRAMA MI TIERRA, MI CASA}

A fines del año 2011, el Gobierno Provincial de Santa Fe creó la Secretaría de Estado del Hábitat (SEH), una nueva estructura con rango ministerial orientada a promover una política habitacional más amplia e integradora. Esta propone un cambio de paradigma de las políticas públicas tradicionales, normalmente asociado a la entrega de la vivienda "llave en mano", por un concepto que incluye un abanico de soluciones, integrando al ciudadano en el proceso de construcción y promoviendo el esfuerzo compartido de todos los actores sociales (públicos y privados) en el abordaje del problemática habitacional.

El programa emblemático fue "Mi Tierra, Mi Casa", el cual tiene por objetivo principal habilitar un conjunto de herramientas orientadas a garantizar el acceso a suelo urbanizado, lotes con infraestructura y servicios básicos

\footnotetext{
${ }^{1}$ Entrevista realizada al agrimensor e ingeniero civil Raúl Álvarez Director del Servicio Público de la Vivienda, ex - subsecretario de la Secretaria de Planeamiento de la Municipalidad de Rosario, 19/07/17.
} 
a precios accesibles inferiores a los del mercado, para diferentes sectores de la población que no cuenten con un lote o una vivienda propia.

Los equipos de trabajo interdisciplinarios están conformados por un personal especializado en hábitat perteneciente a la SEH y en forma coordinada con las herramientas de la Dirección Provincial de Vivienda y Urbanismo (DPVyU), tomando en consideración el objeto de cada urbanización y la tarea a realizar. Este último organismo históricamente orientado a programas de vivienda para grupos familiares no propietarios, mayoritariamente de clase media y media - baja, financiados por los fondos de FONAVI y de otros programas especiales de la Nación.

Mi Tierra, Mi Casa promueve áreas de intervención en concordancia con planes y directrices locales, identificando y delimitando aquellas que tengan especial interés urbanístico en relación al valor estratégico para las políticas locales relacionadas al hábitat, atendiendo a los procesos de crecimiento y completamiento del tejido urbano existente (Secretaría de Estado del Hábitat, 2012). Es decir que el tema de la vivienda se relaciona directamente con la planificación urbana, con lo que propone cada instrumento de cada localidad, siendo el suelo un recurso patrimonial escaso y estratégico.

El suelo generalmente es propiedad de la provincia o de las localidades. Ambos gobiernos trabajan conjuntamente materializado en un convenio de "gestión asociada de urbanizaciones de interés social". El programa incluye la identificación y captación de suelo urbano, sub-urbano y rural, entendido como el proceso de priorización de tierras de utilidad pública para incorporar al registro provincial de suelo a través de operatorias de donación, adquisición o compra, expropiación, gestión asociada de urbanizaciones y demás operatorias que posibilitan a la SEH disponer de suelo para ser urbanizado. A través del registro provincial el Estado logra identificar y catalogar el suelo privado de valor urbanístico y social al mismo tiempo que calificar, administrar y disponer del suelo de dominio público, siendo un instrumento esencial para la política habitacional.

En este sentido, en el año 2015 la SEH lanzó una convocatoria a tenedores de suelo privado para ampliar la oferta de lotes disponibles, en donde el propietario aporte un área mayor y el Estado Provincial los fondos para su urbanización. El requisito era que el suelo deba ser potencialmente utilizable para la construcción de viviendas, de uso urbano, sub-urbano o rural (reconvertible a urbano), no inundable y con una superficie mayor a $10.000 \mathrm{~m}^{2}$. Esto se realiza a través de un convenio por el cual el terreno pasa a la Provincia, es decir que el Estado no expropia, ni compra el terreno sino que es una asociación público - privada. Una vez que ejecuta las obras de infraestructura la SEH (2015:2) abre dos posibilidades para el propietario: recibir como contraprestación una cierta cantidad de lotes urbanizados en función del valor del área aportada (pudiéndolo vender, retener o desarrollar un emprendimiento) o convenir un esquema comercial sobre el total de los lotes urbanizados, cobrándolo en cuotas a los adjudicatarios. ${ }^{2}$

En suma, el programa conviene la gestión asociada entre entidades públicas y/o con personas físicas o jurídicas privadas poseedores de suelo, conformando "consorcios urbanísticos" entre el Gobierno Provincial y/o los Gobiernos Locales, propietarios privados, instituciones u organizaciones de la sociedad civil, destinados a la ejecución de proyectos de urbanización, asegurando una justa distribución de cargas y beneficios entre todos los propietarios del sector.

La SEH realiza la gestión de su urbanización de los predios identificados en el registro provincial de suelo, abarcando la elaboración de los proyectos técnicos y normativos de subdivisión de suelo, definición de trazados, dotación de infraestructura y servicios básicos, donación de espacios públicos, áreas verdes y reserva de tierras para futuros equipamientos comunitarios a construir por cada municipio. También la asignación de lotes de diferentes tamaños, localizaciones y valores, en condiciones de posesión y titularización segura (Secretaría de Estado del Hábitat, s/f).

El producto final, "lotes urbanizados", se asignan a los beneficiarios a través de convenios específicos con organismos oficiales e instituciones de la sociedad civil o por medio de sorteo público a la demanda general.

\footnotetext{
${ }^{2}$ En tal caso, los primeros en cobrar a partir de las cuotas de los beneficiados por el plan serán los particulares hasta completar el pago del suelo, y luego lo hará la Provincia. Esta segunda posibilidad está siendo aplicada con buenos resultados en la ciudad de Villa Gobernador Gálvez, localizada al sur de Rosario.
} 
Esta última modalidad se realiza a medida que se van generando los terrenos urbanizados a través del Registro Único de Inscripción Permanente (RUIP) de la DPVyU, siendo esta forma de inscripción similar a la de los tradicionales planes de vivienda. ${ }^{3}$

El público al cual está destinado debe ser mayor de edad, constituir un grupo conviviente permanente (santafesino o con residencia en la provincia), de sectores medios y populares con ingresos demostrables, que no tienen casa o lote propio ni haber sido adjudicatarios de otras viviendas financiadas por el Estado.

El beneficiario deberá pagar su lote con un anticipo del $15 \%$ al $25 \%$ del total, pudiendo cancelarlo en un plazo de 3 a 6 meses y el resto en cuotas sin interés. El plan de pagos podrá ser a 12, 24, 36, 48 o 60 meses, lo cual será fijado en función de los salarios del grupo, no pudiendo superar el $20 \%$ del total de los ingresos.

El precio del lote varía según su tamaño y ubicación en la urbanización, teniendo los emprendimientos distintos precios en la ciudad. Al respecto Lauría directora de SEH Santa Fe en el programa televisivo CTV Cifras (2013) expresa que:

“... el precio de venta de los terrenos es muy inferior a los precios que se encuentran en el mercado, aproximadamente un $50 \%$ menos (...). Este precio accesible es por el subsidio del Estado, fundamentalmente en el valor que tiene la tierra, estamos prácticamente poniendo en el mercado un lote al valor del costo de infraestructura que está llevando adelante el Estado".

Una vez abonado dicho anticipo, la DPVyU entrega al beneficiario el boleto de compra venta y el dominio del terreno. La escrituración del lote y el cercado perimetral lo realiza el adjudicatario, debiendo empezar a construir a partir de 6 a 12 meses de haber firmado el boleto de posesión. La construcción dependerá de cada familia, es decir es una etapa de autogestión, pudiendo llevar adelante un proyecto independiente o elegir un modelo de casa entre los prototipos diseñados por la DPVyU.

Los que elijan la segunda alternativa también podrán acceder al "Crédito Personal Banco Municipal" para la compra de materiales, no siendo obligatorio dado que los adjudicatarios podrían costear ellos mismos los materiales, dentro del plazo exigido para construir y habitar. En caso de elegirlo, participan de un taller en donde escogen el modelo de vivienda que mejor se adapta a sus necesidades sobre una serie de propuestas que pone a disposición el Gobierno Provincial. Además, el secretario de la SEH Leone indicó en el diario Mirador Provincial (2017) que:

“... se entrega a las familias un manual de uso y mantenimiento de la vivienda. Se trata de un instructivo útil que aborda el cuidado, mantenimiento y uso adecuado de los inmuebles, consejos útiles sobre aspectos prácticos y legales, y valiosas propuestas que contribuyen a prolongar la vida útil de la vivienda, el uso eficiente de la energía y el cuidado de medio ambiente".

Una vez con el prototipo seleccionado pueden acceder al crédito cubriendo un monto en materiales de hasta 75.000 pesos en el año 2013 y 150.000 pesos en el año 2015, con un plazo de devolución de 120 meses, a una tasa de interés fija en pesos del 12\% los primeros años (Gobierno de Santa Fe, 2014).

Asimismo, los lotes urbanizados se constituyen en el insumo base de otros programas de viviendas unifamiliares o colectivas que impulse el Gobierno Provincial, sea a través de la SEH o de la DPVyU, como ser el de "Construcción y/o Financiamiento de Soluciones Habitacionales", y el Gobierno nacional a través del Ministerio del Interior, Obra Pública y Vivienda, como el "Programa de Crédito Argentino" (Pro.Cre.Ar.) hasta el año 2015 y luego con el "Programa Federal de Construcción de Viviendas - Techo Digno" (PFCV). También de créditos privados como el caso de la "Cooperativa de Viviendas" en la ciudad de Rosario.

\footnotetext{
${ }^{3}$ El Ruip es administrado por los gobiernos locales, excepto en las ciudades de mayor tamaño de la provincia (Rosario y Santa Fe) en donde hay oficinas de la Dirección Provincial de Vivienda y Urbanismo. Los sorteos son públicos y gratuitos, con la presencia de escribanos, y se realizan a través de la Caja de Asistencia Social de la Provincia - Lotería de Santa Fe. Además, de la participación a través de sorteo, la posterior comprobación de la declaración jurada son condiciones exclusivas para que el grupo familiar pueda acceder al lote propio.
} 


\section{ALCANCE EN LA CIUDAD DE ROSARIO}

En el año 2012, el Consejo Municipal de Rosario aprobó la ordenanza № 8.976 de urbanización con dos proyectos para el programa: "Loteo Avellaneda Oeste" y "Parque Habitacional Ibarlucea", en donde se inscribieron aproximadamente 4.500 familias. Rosario fue la primera ciudad en instituir Mi Tierra, Mi Casa y luego se extendió al resto de la provincia de Santa Fe.

El primero está en el Distrito Norte, entre las calles Cullen - Ugarte, Tres Sargentos, límite del Municipio y 1326, cuenta con 66,2 hectáreas de extensión. En una primera etapa, la urbanización conto con 1.033 lotes, de un total de 1.420 , entre 200 y $350 \mathrm{~m}^{2}$, a un costo de entre 50.000 y 60.000 pesos. Las tareas a cargo de Edeca SA comenzaron en mayo del año 2013 y finalizaron el 2015.

El segundo se encuentra en el Distrito Sudoeste, comprendido entre la avenida Cisneros, calles More, Maradona, Medina, 1821 y 1886 en un predio de 18,5 hectáreas. Allí se habilitaron 580 lotes con una superficie que va de los 150 a $180 \mathrm{~m}^{2}$, construidos entre los años 2013 y 2015 por Dycasa SA y Epreco SA. Los valores de los terrenos fueron menores, oscilando entre 30.000 y 40.000 pesos.

En el año 2015, se aprueba la ordenanza № 9.481 del Plan Especial ARE № 10 "Parque Habitacional Ibarlucea Este" en donde destina el Sector 2, conocido como la "Ex villa Olímpica", para la provisión de lotes del Programa. Esta tercera urbanización del Programa, al igual que la primera se localiza en el Distrito Norte. Está comprendida entre las calles Cullen y Ugarte, 1379, Salvat, Blomberg, Mocoreta, 13.109 y Maldonado. Las obras comenzaron en el año 2016 con la urbanización de 470 lotes por las empresas Brajkovic SA y Milicic SA., teniendo previsto un total de 1200 lotes.

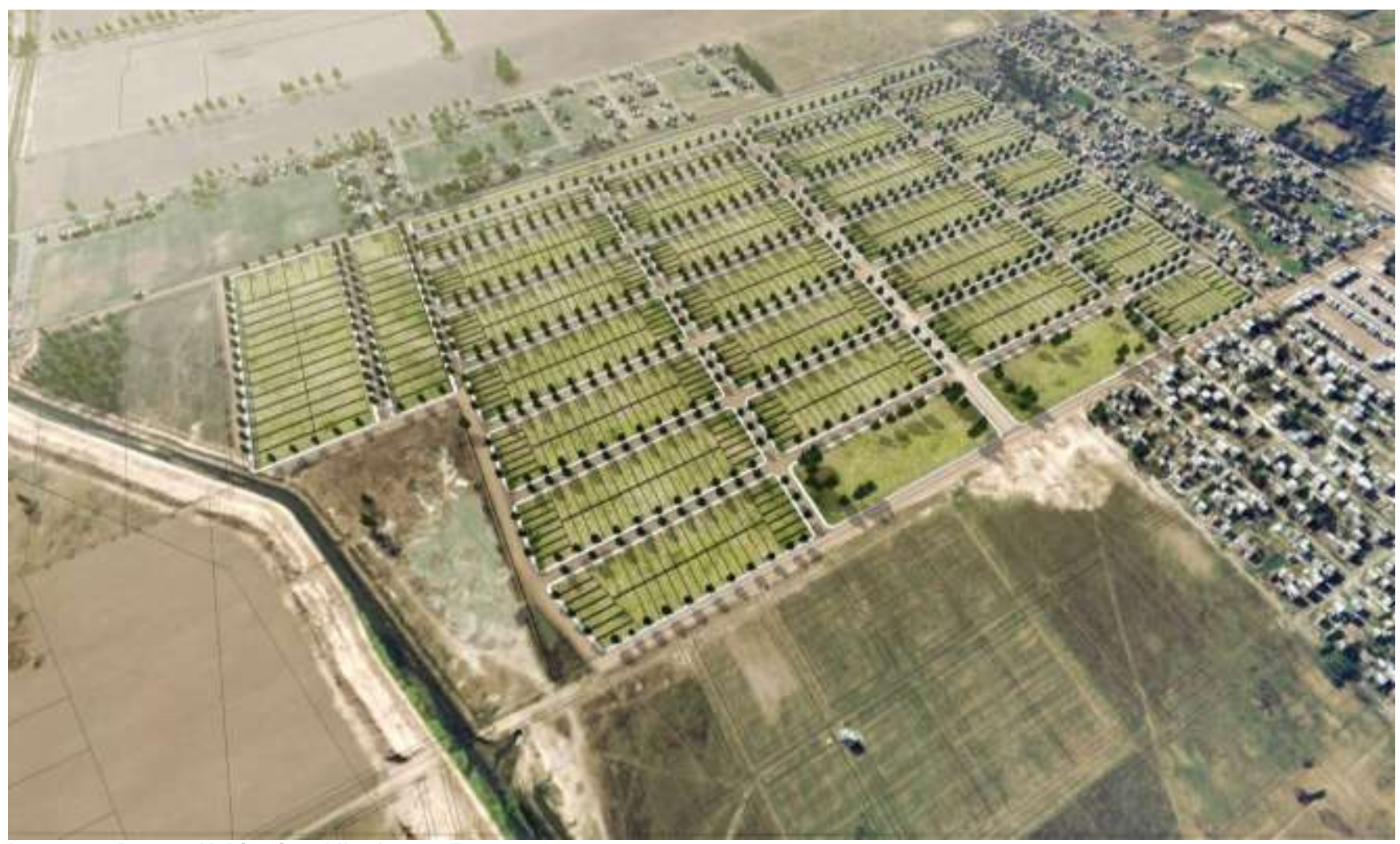

Imagen 1. Parque Habitacional Ibarlucea: Etapa 1

Fuente: La Capital, 2013. 


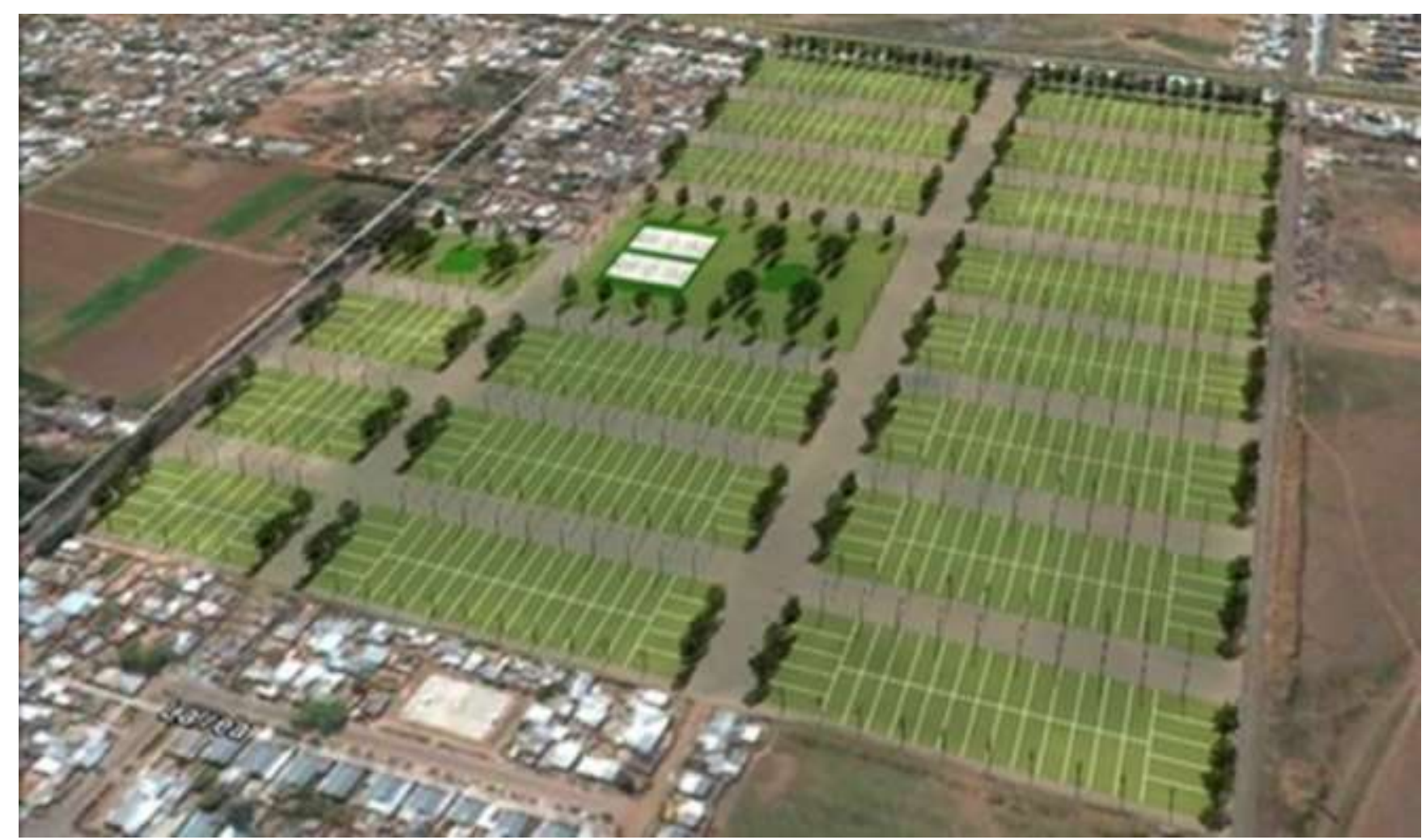

Imagen 2. Loteo Avellaneda Oeste

Fuente: Sin Mordaza, 2012. 


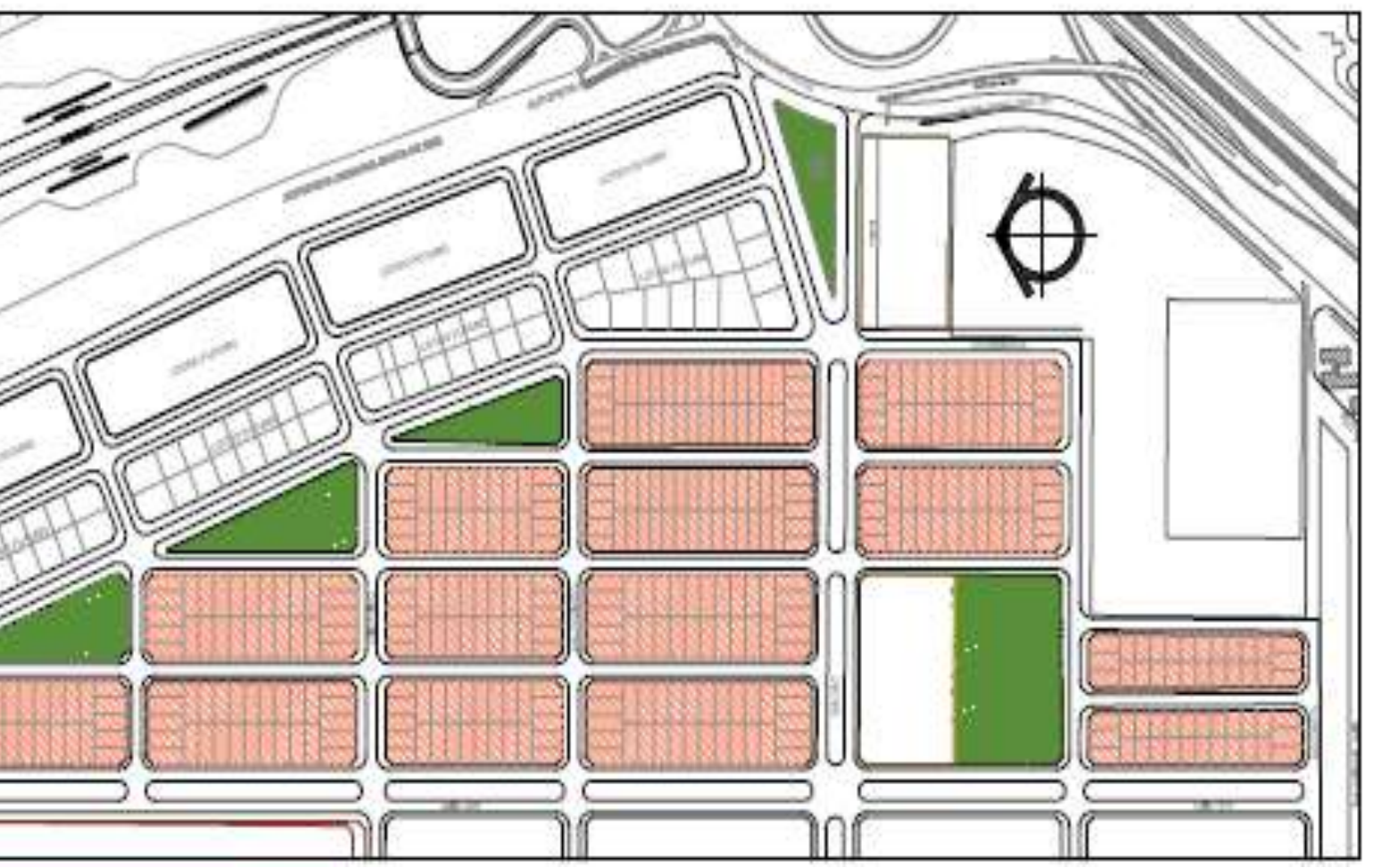

Imagen 3. Urbanización Ex Villa Olímpica

Fuente: Pliego Licitación Gobierno de Santa Fe, 2016.

La elección del sitio a urbanizar en la ciudad se relacionó con la disponibilidad de tierra de interés público para vivienda visualizada en el Registro Provincial de Suelo, en concordancia con los lineamientos del Plan Urbano Rosario, atendiendo a los procesos de crecimiento y completamiento urbano, y también con la coexistencia de una buena conectividad. Fernández de la SEH menciona que "... sin conectividad no hay urbanización por eso se localizan sobre avenidas o autopistas. Trabajando en forma conjunta la SEH con el Municipio y el Plan Urbano Rosario, integrándose y completando el tejido urbano existente". ${ }^{4}$

\footnotetext{
${ }^{4}$ Entrevista realizada a la subsecretaria de Planificación y Gestión de la Secretaria de Estado del Hábitat Arq. María Laura Fernández. Rosario, 1/02/18.
} 
El Parque Habitacional Ibarlucea y la urbanización Ex Villa Olímpica son parte del "Plan Habitacional Rosario Norte". Estas poseen una mejor localización que el segundo desarrollo y ubicándose entre ambas la "Zona Cero" (reciente barrio de vivienda social que cuenta con espacios verdes, centro de salud, jardín de infantes, escuela primaria y secundaria), rodeadas por un club de rugby, barrios de clase media-baja y algunas pocas áreas rurales, contiguo a la Avenida de Circunvalación, Autopista Rosario - Santa Fe y Ruta Nacional 34. Contrariamente el Loteo Avellaneda Oeste esta bordeado por distintos asentamientos irregulares y áreas rurales, lindante con la Avenida Avellaneda, careciendo de equipamientos y servicios.

En cuanto al reparto de lotes y créditos, se distribuye un $60 \%$ en relación al padrón general (previsto $10 \%$ discapacitados y $10 \%$ para fuerzas de seguridad), $20 \%$ para sindicatos y otro $20 \%$ para trabajadores de empresas radicadas en el lugar de adjudicación (SL24, 2012).

Todos los emprendimientos cuentan con suministro de: agua potable, energía eléctrica, alumbrado público, desagües pluviales, pozo absorbente, arbolado público, cordón cuneta, estabilizado granular para todas las calles y pavimento en aquellas arterias donde circule el transporte público, demandaron una inversión del Gobierno Provincial de 112 millones de pesos. También se amplían los servicios de recolección de basura y las líneas de transporte público.

El porcentaje de la superficie que se destina para la apertura de calles, la creación de espacios verdes públicos y los sitios para la construcción de los equipamientos comunitarios son distintos: el Parque Habitacional Ibarlucea posee un $38 \%$ y el Loteo Avellaneda Oeste un 48\%. Cabe señalar que la construcción de los espacios comunitarios está a cargo del Municipio, realizándose de acuerdo a lo que prevé y necesita el nuevo emprendimiento referido a la salud, la educación, la seguridad, el deporte, lo cultural, entre otras actividades. Al respecto Fernández explica que:

“.... no siempre es lo mismo en cada urbanización cuando son familias jóvenes con hijos, generalmente hay un centro asistencial y una escuela. Por ejemplo el Parque Habitacional Ibarlucea, que es parte de un plan especial mayor, se vincula como un todo con la Zona Cero que es el centro. Allí ya hay un jardín de infantes, escuela primaria y secundaria, centro de salud, por eso el espacio destinado para equipamientos fue menor". ${ }^{5}$

El tipo y magnitud de estas obras amplían la oferta de equipamientos e infraestructura de la ciudad, habilitando de esta forma, mayor cantidad de suelo urbanizado. Esto da cuenta de la complejidad de la actuación en la que participan numerosos actores públicos y privados (empresas prestatarias de servicio, varias reparticiones del Gobierno Provincial y de la Municipalidad de Rosario, empresas contratistas privadas), con preocupaciones y dinámicas diferentes.

La construcción de las viviendas implico re-estudiar cada uno de los aspectos que intervienen en ese proceso. En este sentido se abordó el concepto de "universalidad aplicado al desarrollo del hábitat", dado que no todas las familias son iguales, por el contrario cada una tiene sus características particulares, no solo en el momento de recibir la vivienda sino también hacia el futuro. Por ello se hizo referencia a un proyecto inclusivo, pudiéndose adaptar a cada situación e incorporando al individuo en la diversidad y complejidad de sus necesidades, capacidades y aspiraciones (discapacitados, ancianos, niños).

Además, la flexibilidad tipológica permite múltiples variables de crecimiento del grupo familiar (adhesión de habitaciones, baños, etc.) y funciones complementarias a la vivienda que forman parte de la realidad socioeconómica de la sociedad (incorpora local comercial, taller, estudio u otro espacio vinculado al trabajo). La diversidad de su materialización (tipológica y formal) se vinculó con los recursos naturales, económicos y humanos disponibles del lugar y con la posibilidad de autoconstrucción. El aprovechamiento y utilización de energías renovables interviene en la modificación de los patrones de consumo y producción energética, orientados al beneficio del ambiente (Secretaría de Estado del Hábitat, s/f: 20-21).

El núcleo básico posee una superficie de $30 \mathrm{~m}^{2}$ (baño, cocina, lavadero y estar comedor). De acuerdo a la ubicación en el terreno se determinan las distintas variables, pudiendo crecer la vivienda de manera: lineal,

\footnotetext{
5 Ídem 4.
} 
compacta o en altura. Teniendo en cuenta las características y proyectos de cada familia, se pueden construir nuevos espacios, incorporando uno o dos dormitorios, siendo extensible a futuras ampliaciones.
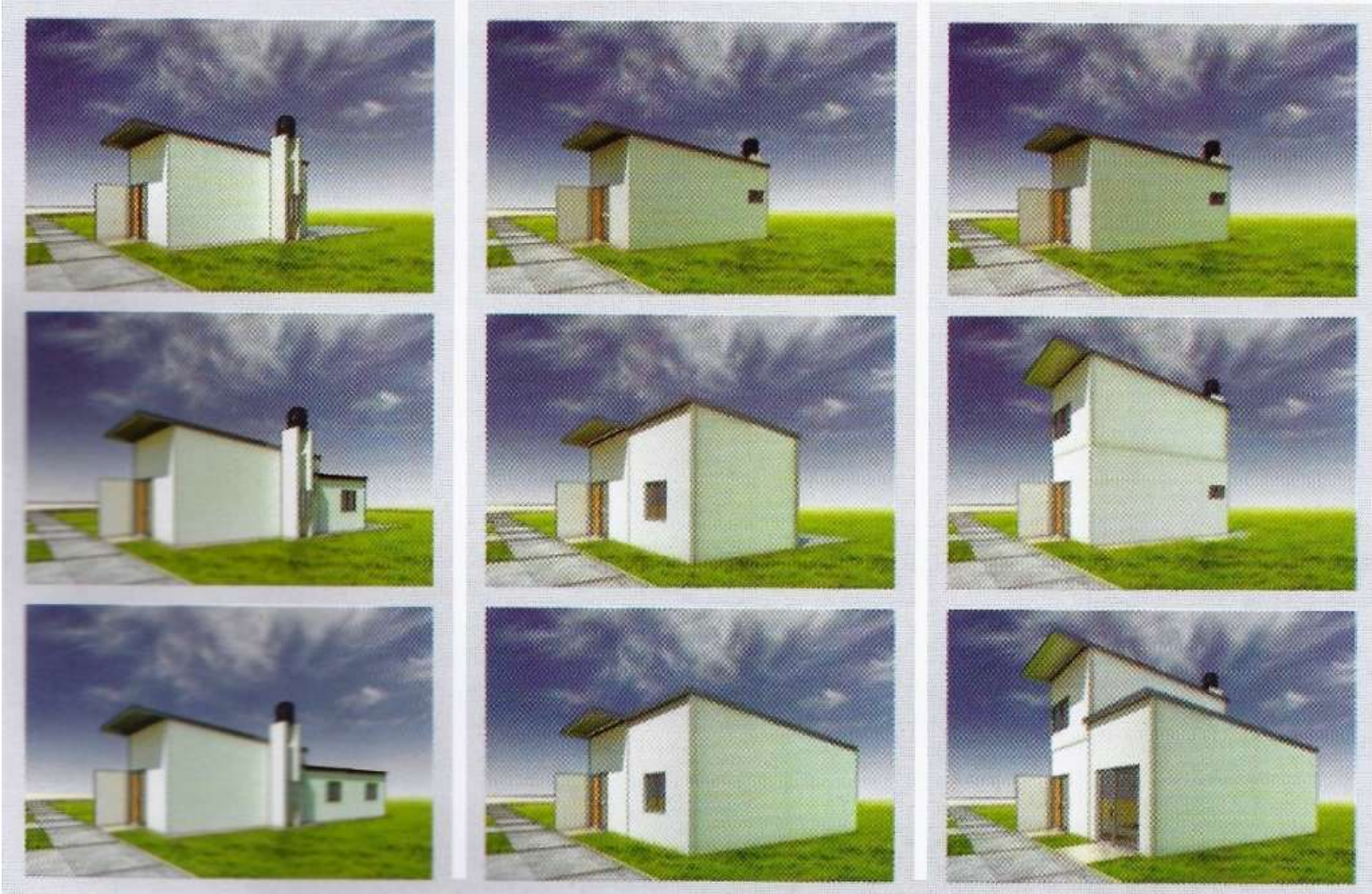

Imagen 4. Posibilidades de construcción del prototipo universal

Fuente: Secretaría de Estado del Hábitat, s/f.

Los diferentes conjuntos presentan una densidad media o baja (de planta baja hasta dos pisos) que no estimula la propiedad horizontal por los conflictos que se han producido anteriormente en viviendas sociales para asegurar su mantenimiento.

La construcción de las viviendas en el "Parque Habitacional Ibarlucea" y el "Loteo Avellaneda Oeste" mayoritariamente fue llevada adelante por las familias con un proyecto independiente, utilizando el prototipo diseñados por la DPVyU. La SEH solo construyo 32 viviendas para la policía en la segunda urbanización.

No obstante desde mediados del año 2016, en el "Parque Habitacional Ibarlucea" y en la "Ex Villa Olímpica" se están construyendo viviendas, también con los prototipos universales (260 y 360 unidades respectivamente, destinadas a la demanda general). Las obras se dividen en cinco proyectos a cargo de las empresas Dinale SA, Dycasa Sam Epreco SRL y Fega-Sanimat, financiados con el PFCV -Techo Digno. Estas son las primeras viviendas que interviene el Gobierno Nacional después de ocho años sin planes federales en la provincia. De acuerdo con el diario La Capital (2016): “... el esquema sellado en esta oportunidad es que el 66 por ciento (430 millones de pesos) por Nación y el 34 por ciento por la provincia (que dispuso del suelo y de la infraestructura)". 


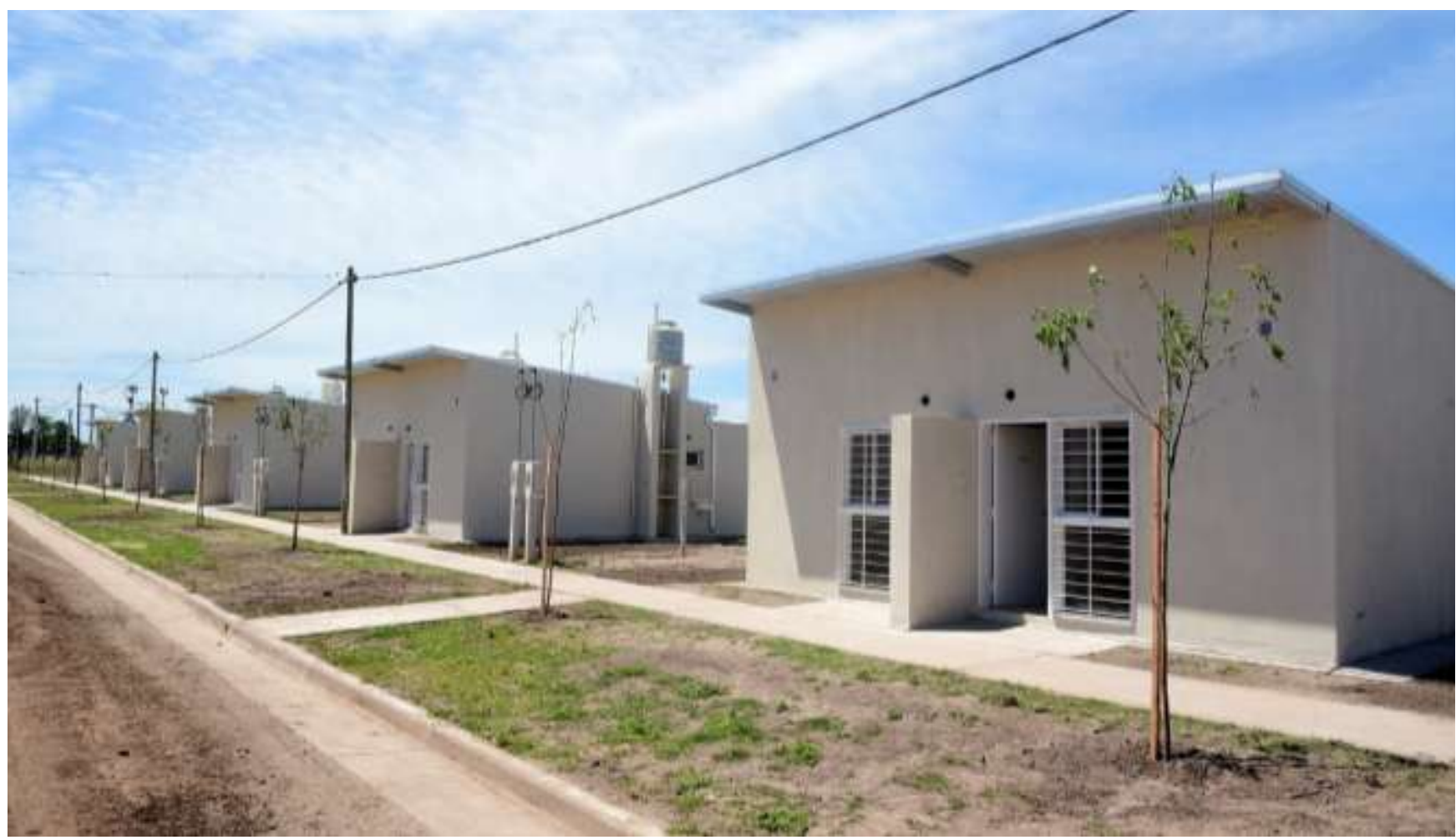

Imagen 5. Viviendas en Parque Habitacional Ibarlucea

Fuente: El ciudadano, 2016.

En recapitulación desde finales del año 2015, la Secretaría de Estado del Hábitat amplio sus tareas ya no solo asumiendo la captación de suelo y definición de proyectos de urbanización (con infraestructura y servicios), acercando a los beneficiarios el derecho a la ciudad, sino que también en la construcción de vivienda a través de financiamiento público, intentando materializar el derecho a la vivienda. Al respecto el gobernador Lifschitz expreso en el diario La Capital (2016) que:

"La política de vivienda no es una política cualquiera, sino que es estratégica, y apunta al corazón de la exclusión social con el fin de integrar a muchos ciudadanos a la comunidad (...). La falta de vivienda es el primer elemento de discriminación y exclusión, por eso es fundamental, además de generar trabajo y mejorar los servicios, que podamos como Estado garantizarle a cada familia un techo propio digno".

Además, el gobernador explicó en el mismo medio que: "construir viviendas sociales genera trabajo, porque implica tres o cuatro puestos de trabajo por unidad, es decir que con la ejecución de estas 630 que estamos firmando, habrá dos mil familias que tendrán asegurado su sustento, por lo menos por el tiempo que duren estas obras".

De acuerdo a los registros en el año 2018 el sistema lleva inscripto 20.558 postulantes en la ciudad de Rosario, de los cuales 18.952 solicitan viviendas, mientras que 1.596 demandan lotes con infraestructuras y servicios pertenecientes al programa provincial Mi tierra, Mi casa (Mirador Provincial, 2018). 


\section{EVALUACIÓN DE LOS CAMBIOS Y LAS ACCIONES EJECUTADAS DURANTE LA GESTIÓN}

El Programa "Mi Tierra, Mi Casa" se implementó en las localidades con mayor déficit habitacional provincial y con disponibilidad de áreas de intervención, según los planes y directrices locales. En Rosario, como en otras ciudades por ejemplo Santa Fe, Rafaela, entre otras, la localización de las urbanizaciones fue en los bordes urbanos. A pesar de tener una buena conectividad y completar el tejido urbano existente, afecta a los destinatarios, ya que siempre se encuentran alejados del centro y en derivación de los principales servicios y equipamientos que ofrece. Estas grandes operaciones unitarias si bien facilitan el acceso al suelo y a la vivienda, no contribuyen a la diversificación socioespacial de la ciudad.

Además, se identifican particularidades en las distintas ciudades de la provincia de Santa Fe. Al respecto Fernández explica que: “... esto tiene que ver con el acceso a otros programas que tuvo y pudo coordinar cada localidad".

En la ciudad de Rosario, la Secretaria de Estado de Hábitat, en sus inicios hasta el año 2015, se abocaba solo a la urbanización y ofrecía para la compra de materiales el "Crédito Personal Banco Municipal Rosario". La mayoría de las viviendas, por ejemplo en el caso del Parque Habitacional Ibarlucea se construyeron por autogestión financiadas con el "Pro.Cre.Ar". Luego, desde el año 2016, al no poder acceder los beneficiarios también a este crédito, la SEH incorporó a la urbanización original la construcción de viviendas con el financiamiento nacional del "PFCV -Techo Digno".

Es decir que a lo largo de los siete años de gestión, se pueden identificar dos etapas fuertemente marcadas, coincidentes con las políticas habitacionales nacionales y los cambios de gobierno en Argentina. La SEH es un organismo público flexible que intenta dar a la propiedad un rol social y no económico, regulando el uso y el precio del suelo urbano.

En una primera etapa (2012 - 2015) intenta cumplir con el derecho a la ciudad, ampliando la infraestructura básica y los servicios urbanos, como así también destinando espacio para el equipamiento comunitario, distribuidos con criterios equitativos y en base a las necesidades del sector. Cabe señalar que la tarea conjunta con las tres empresas prestatarias de servicio involucrado (Empresa Provincial de la Energía, Aguas Santafesinas SA y Litoral Gas) y debido a la dimensión de las obras, involucró en ciertas instancias reuniones y fiscalización de técnicos de la Municipalidad de Rosario.

En la segunda etapa (2016 - 2018), con financiamiento nacional, se hace cargo de materializar el derecho a la vivienda digna. Además, el diseño de la vivienda universal, agrega Fernández: “... implicó adaptar la política habitacional con otras políticas del gobierno provincial, de orden ambiental, como ser el aprovechamiento y utilización de energías renovables incorporando tanques eléctricos, entre otros elementos vinculados al hábitat sostenible". 7

En relación a los avances y las posibilidades de cada uno de los adjudicatarios de conseguir el terreno y la vivienda, surgieron algunas críticas a través de los medios de comunicación locales. En el diario digital Sin Mordaza menciona el diputado provincial Toniolli sobre el Parque Habitacional Ibarlucea y Loteo Avellaneda Oeste que:

“... el proceso de urbanización de las tierras no ha avanzado, y el acceso al crédito que el programa debía garantizar, tanto para el terreno como para el proyecto de construcción, presenta serias dificultades y condiciones no estipuladas previamente que son difíciles de cumplimentar. Se exige a los adjudicatarios un anticipo del \% 25, la mensura y cercado del terreno y la construcción de la totalidad de la vivienda en el plazo de un año, como requisitos para el acceso al crédito" (Sin Mordaza, 2015:1).

Por último, la evolución inflacionaria de la economía presento otra problemática. Esta afectó directamente el valor de los materiales y de la mano de obra de la construcción, requiriendo una actualización en el monto total

\footnotetext{
6 ídem 4.

7 Ídem 4.
} 
de los créditos entregados vinculados con "Mi Tierra, Mi Casa". Por ejemplo los "Créditos Personal del Banco Municipal" para la compra de materiales que se articulan con el programa se iniciaron en el año 2013 con un monto de hasta 75.000 pesos incrementándose hasta 150.000 pesos para el 2015 , año en que deja de funcionar.

\section{REFLEXION FINAL}

El desafío del gobierno provincial radicó en elaborar una política pública capaz de brindar a la clase media y media-baja el acceso efectivo a un lote urbanizado. Con este fin, la Secretaria de Estado del Hábitat implementa el Programa "Mi Tierra, Mi Casa" que va completando con infraestructura, servicios y áreas para equipamientos los terrenos vacantes (públicos y/o privados) de la ciudad, a precios inferiores a los que ofrece el mercado.

La implementación del mismo no genero procesos especulativos, usuales en otros programas de financiamiento en donde el crédito es para la compra del terreno en el libre mercado. Al ser el Estado el que compra, subdivide, urbaniza y vende los lotes, este es quien le asigna el valor al suelo. Esto no quiere decir que la provisión de infraestructura, servicios y equipamientos en áreas pocos consolidadas no den lugar a aumentar el valor y el desarrollo inmobiliario en los lugares obteniendo sobreganacias sino que el Estado como agente productor de suelo urbano logra regularlo en pos de que la población pueda acceder a su terreno y posterior vivienda.

Los proyectos poseen una buena conectividad, ubicados sobre avenidas existentes, dentro de la periferia rosarina. A pesar de que se dotan de nuevas infraestructura, extienden los servicios y se destina espacios para la construcción de equipamientos urbanos, estos son insuficientes. Esto es debido a la poca cantidad ejecutada hasta el momento (2.470 lotes y 652 viviendas) en relación a la demanda real (18.952 viviendas y 1.596 lotes), excediendo la problemática habitacional a la esfera provincial, y la ubicación establecida en los bordes urbanos, excluyendo el acceso de las personas de menores ingresos a las ventajas que ofrece vivir en el interior de la ciudad, no contribuyendo a la diversificación y equidad socioespacial.

Las grandes operaciones unitarias evidencian las limitaciones del gobierno para conseguir terrenos mejores ubicados, coincidiendo con las políticas públicas aplicadas en las últimas décadas, en donde se privilegian el número de viviendas sobre la localización de las nuevas urbanizaciones. Tal vez podría pensarse en la distribución de terrenos de menor tamaño de forma más dispersa e integrada con la ciudad y no todos juntos, en tres grandes áreas de Rosario. Esto requiere un cambio profundo en el proceso de toma de decisiones técnico políticas que como todo proceso social, llevará un tiempo considerable.

Por último, la construcción de viviendas, principalmente en los casos de la autoconstrucción contribuye con la generación de empleo e incentivan la industria de la construcción local. El crecimiento de los puestos de trabajo en este rubro, por lo menos tres o cuatro obreros permanentes por unidad, y de otros sectores vinculados es significativo. Mi Tierra, Mi Casa representa un impulso para el mercado interno, que incentiva a las empresas locales y favorece la industria regional. Por ello, la clave del Programa es financiar la construcción de nuevas viviendas.

En contraparte, la evolución inflacionaria de la economía desde el año 2012 al 2018, afectó directamente el valor de los materiales y la mano de obra de la construcción. Si bien el crédito para cubrir el monto de los primeros se fue actualizando cuando estaba vigente el "Crédito Personal del Banco Municipal", resulta cada vez más difícil cubrir el monto total de la construcción de la vivienda. 


\section{BIBLIOGRAFÍA}

\section{Libros y revistas:}

BARENBOIM, C. A. (2014). Mercado inmobiliario, normativa e impacto territorial: Rosario y su Periferia. Rosario: Editorial UNR.

(2017). La problemática de la vivienda y la persistencia del déficit habitacional: el caso de la ciudad de Rosario, Argentina. Revista Papeles de Coyuntura (Bogotá), 43, 128 - 153.

CELADE. (2010). Conceptos principales atinentes al déficit habitacional. Washington: ONU.

CENTRO DE ESTUDIOS ECONÓMICO Y SOCIALES SCALABRINI ORTIZ. (2016). Situación del mercado viviendas Rosario. CESO: Santa Fe.

SECRETARÍA DE ESTADO DEL HÁBITAT. (s/f). Herramientas para una política de Hábitat y Vivienda. Santa Fe: Gobierno de Santa Fe.

2015. Llamado a presentación de Propuestas Para La Gestión Asociada De Urbanizaciones En Terrenos Aptos Para Loteos. Santa Fe: Gobierno de Santa Fe.

SCIARA, A. J. (2011). Lineamientos para una política del hábitat y urbanismo. Rosario: Consejo Económico y Social.

\section{Actas, ordenanzas y resoluciones:}

CONSEJO ECONÓMICO Y SOCIAL. (2010). Actas de reunión Comisión Hábitat y Asentamientos Irregulares. Rosario: Municipalidad de Rosario

CONCEJO MUNICIPAL. (2012). Ordenanza № 8976 Plan Especial Programa Mi Tierra, Mi Casa. Parque Habitacional Ibarlucea y Parque Habitacional Avellaneda Oeste. Rosario: Municipalidad de Rosario.

. (2015). Ordenanza № 9481 Plan Especial Parque Habitacional Ibarlucea Este. Rosario: Municipalidad de Rosario.

SECRETARÍA DE ESTADO DEL HÁBITAT. (2012). Resolución № 153 de creación de Programa Mi Tierra, Mi casa. Santa Fe: Legislatura de la provincia de Santa Fe.

\section{Fuentes electrónicas:}

https://www.lacapital.com.ar/la-ciudad/provincia-y-nacion-haran-630-viviendas-rosario-n1246816.html (Consulta 15/02/18).

https://www.santafe.gov.ar/noticias/noticia/210744 (Consulta: 20/01/18).

http://www.sinmordaza.com/noticia/302392-denuncian-irregularidades-del-plan-mi-tierra-mi-casa.html (Consulta 25/01/18).

https://sl24.com.ar/2012/08/punto-por-punto-como-es-el-plan-habitacional-que-anuncio-la-provincia/ (Consulta 27/01/18).

http://www.miradorprovincial.com/?m=interior\&id_um=162768-provincia-entrego-800-viviendas-en-rosario-en2017-habitat (Consulta 14/02/18).

https://www.youtube.com/watch?v=dCi6ilGIJ6M (Consulta 10/02/18). 